\title{
Molecular Laser Spectroscopy as a Tool for Gas Analysis Applications
}

\author{
Javis Anyangwe Nwaboh, ${ }^{1}$ Thibault Desbois, ${ }^{2}$ Daniele Romanini, ${ }^{3}$ \\ Detlef Schiel, ${ }^{1}$ and Olav Werhahn'
}

${ }^{1}$ Physikalisch-Technische Bundesanstalt (PTB), Bundesallee 100, 38116 Braunschweig, Germany

${ }^{2}$ Floralis, Filiale de l'Université Joseph Fourier, Grenoble, 6 allée de Bethléem, 38610 Gières, France

${ }^{3}$ 3LIPhy-Laboratory of Interdisciplinary Physics (formerly LSP), CNRS Unité Mixte de Recherche 5588, 140 rue de la Physique, Batiment E45 Université J. Fourier de Grenoble 38402 St Martin d'Hères, France

Correspondence should be addressed to Javis Anyangwe Nwaboh, javis.nwaboh@ptb.de

Received 25 January 2011; Accepted 28 March 2011

Academic Editor: Veronica Vaida

Copyright (C 2011 Javis Anyangwe Nwaboh et al. This is an open access article distributed under the Creative Commons Attribution License, which permits unrestricted use, distribution, and reproduction in any medium, provided the original work is properly cited.

\begin{abstract}
We have used the traceable infrared laser spectrometric amount fraction measurement (TILSAM) method to perform absolute concentration measurements of molecular species using three laser spectroscopic techniques. We report results performed by tunable diode laser absorption spectroscopy (TDLAS), quantum cascade laser absorption spectroscopy (QCLAS), and cavity ring down spectroscopy (CRDS), all based on the TILSAM methodology. The measured results of the different spectroscopic techniques are in agreement with respective gravimetric values, showing that the TILSAM method is feasible with all different techniques. We emphasize the data quality objectives given by traceability issues and uncertainty analyses.
\end{abstract}

\section{Introduction}

Throughout the last years many molecular laser spectroscopic techniques have been used to qualify and quantify different physical mechanisms taking place in atoms or molecules [1-7]. Molecular spectroscopy as performed by probing intra- and intermolecular vibrational transitions and further underlying rotational substructure has been used to study and illuminate bond structures and formation of atomic and molecular agglomerates and clusters $[8,9]$.

These techniques are applied due to the absorption, emission, or scattering of electromagnetic radiation by atoms or molecules. The choice of each of these physical phenomena, for example, absorption, for molecular species quantification or qualification, depends on the intended application. Absorption spectroscopy, for instance, is employed to identify and quantify molecular species in gas analysis applications such as remote sensing, atmospheric monitoring, vehicle exhaust emissions, or even exhaled breath gas tests [10-28].

In metrology, molecular absorption spectroscopy could be used to assign amount fractions (concentrations) to species in gas mixtures of known molecular constituents (Note, for a clarification of different terms in use see e.g.: http://goldbook.iupac.org/A00296.html). The determination of the amount fraction of a species without the use of calibrated reference gas mixtures, leads to the socalled "calibration-free" infrared spectrometry. Calibrationfree means the amount of substance fraction of a species is measured in terms of the International System of Units (SI) derived unit $\mathrm{mol} \cdot \mathrm{mol}^{-1}$ without referencing to a standard or a measurement expressed in the same unit [29]. The desire to derive amount fraction results by means of spectroscopy that are directly traceable to the SI triggered the idea of a traceable infrared laser spectrometric amount fraction measurement (TILSAM) method [29, 30].

In order to apply the TILSAM method, typically, a sufficiently resolved, spectrally isolated molecular rovibrational absorption line is preferable. Regarding the derivation of an amount fraction, the documentary description of TILSAM [29] describes the raw data processing, the modeling, the influence quantities, and so forth, based on tunable diode laser absorption spectroscopy (TDLAS). However, 


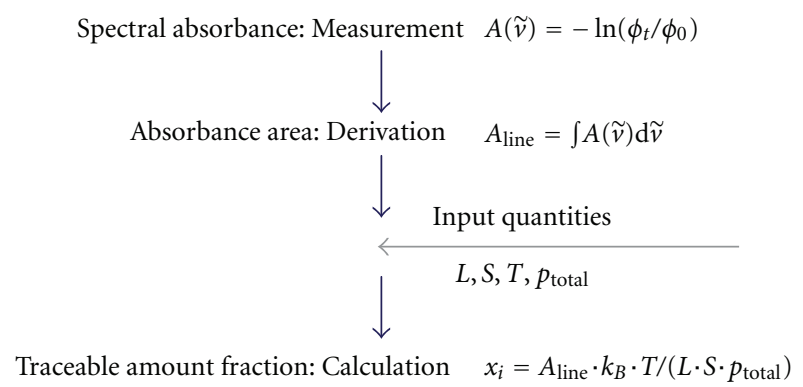

Figure 1: Process of the amount fraction determination according to TILSAM [21], schematic view for TDLAS/QCLAS.

other spectrometric techniques, such as quantum cascade laser absorption spectroscopy (QCLAS) or cavity ring-down spectroscopy (CRDS), can also be used to perform absolute amount fraction measurements based on TILSAM. The TILSAM method can be applied to perform laser spectrometric amount fraction measurements in metrological environments and in field applications $[31,32]$.

Figure 1 visualizes schematically the amount fraction determination due to the TILSAM method. Summarized to four steps, there are just the measurement process, the line area derivation, the incorporation of the input quantities, and the computation of the model equation to subsequently achieve a traceable amount fraction result. The uncertainty of the resulting amount fraction has to be reported according to the ISO GUM [33]. However, the determined amount fraction would be traceable if all input quantities were traceable to the SI units, which unfortunately, to the best of our knowledge, has been achieved very rarely. Although not new in its single steps, the TILSAM method aims to represent and document a combination of steps necessary to apply IR spectrometry as an absolute method. There has been other groups and other termini dealing with this idea. From a metrology point of view, TILSAM promotes these ideas on some standardized basis, providing some descriptive framework and being as flexible as possible to different experimental implementations. Referring to Figure 1 and depending on the spectrometric technique applied, the measurements yield spectral absorbances or absorption coefficients as is the case for TDLAS/QCLAS and CRDS, respectively. Then, line areas are determined either in units of $\mathrm{cm}^{-1}$ for TDLAS/QCLAS or $\mathrm{cm}^{-2}$ for CRDS by means of direct numerical integration or by means of fitting an appropriate line profile to the measured data. Crucial to TILSAM is the subsequent incorporation of the input quantities because, here, traceability of all parameters would be desired to maintain the quality issue of traceability to the final amount fraction result.

Among the most indispensable input quantities, there is the molecular line strength of the probed transition. Line strength values could be taken from data bases like HITRAN [34] and GEISA [35]. These data bases provide valuable information. Among them, there are some lines with incredibly small reported uncertainties, that is, "error codes" [34], based on very sophisticated measurements.
However, there are a larger number of lines as well, where the uncertainties, for example, those of $S$ are somewhat larger and more imprecise; uncertainty budgets in accordance with the ISO GUM [33] are generally not provided. This last point prevents any spectrometrically measured amount fraction based on HITRAN or GEISA from being traceable. As a consequence, a couple of studies have been undertaken to report line strength values and associated GUM compliant uncertainty figures [36, 37]. Recently in the EMRP call 2010 [38], there was a proposal to establish a new reference line data measurement program. The lack of reference data that are more precise than those typically provided by HITRAN and GEISA and which are equipped with quality flags in terms of GUM-compliant uncertainties had also been reported earlier [39]. As reported in [39], for instance, the accurate determination of the uncertainties of the spectrometric data is often suspect or even completely absent, suggesting the need for a precise quantification of the uncertainties associated with the reported spectrometric parameters in [34]. Despite all above, it should be noted here, that HITRAN, GEISA, and other line parameter sources provide absolutely valuable information to any spectroscopist, no matter whether in academic research environments or metrology community. The present work itself takes advantage of the use of HITRAN's beneficials, that is, from line strength data on $\mathrm{CO}$ and $\mathrm{CO}_{2}$ that are reliable within their specified error ranges.

Nevertheless, absolute amount fraction measurements sometimes meet other specific challenges depending on which spectroscopic technique is used. For instance, in intrapulse mode QCLAS, an effect reported by [1] known as rapid passage is observed in the measured absorption signals of molecular species at low pressure. Rapid passage occurs when the laser frequency sweep through an absorption line is faster than the collisionally induced relaxation [1]. This results in an asymmetric or even inverted or oscillating absorption signal. Such molecular effects have to be investigated and considered for any absolute amount fraction measurement.

Also the temperature dependence of the line strength has to be considered. By convention in spectroscopy, line strength figures are reported at some conventional temperature as, for example, that of HITRAN as $296 \mathrm{~K}, S_{0}$. Because of that, for measurements performed at any other temperature $T$, the line strength has to be transformed to $S_{T}$, for example, applying a certain model that explicitly describes the temperature dependence of S. TILSAM, of course, does not require any specific model. However, we need to emphasize the importance of matching the line strength $S_{0}$ to the actual temperature $T$ by means of any appropriate model, for example, as being used in standard analysis.

Additionally there are isotopic abundance issues hidden in the line strength figure that have to be taken into account. Line strength values are typically reported for a certain abundance of the probed isotopologue. However, for a given mixture, generally the abundance of the probed isotopologue may differ from this norm abundance. Therefore, in principle, one would have to correct the line strength figure by substituting $S=S_{T} \cdot r_{\text {iso }}$, where $r_{\text {iso }}$ is the 
isotopic composition factor, given for example, as $r_{\text {iso }}=$ $x_{12 \mathrm{C} 16 \mathrm{O}} / x_{12 \mathrm{C} 16 \mathrm{OHIT}}$, for a probed ${ }^{12} \mathrm{C}^{16} \mathrm{O}$ excitation, where $x_{12 \mathrm{C} 16 \mathrm{O}}$ and $x_{12 \mathrm{C} 16 \mathrm{OHIT}}$ are the abundances of ${ }^{12} \mathrm{C}^{16} \mathrm{O}$ in the sample and the conventional value given by HITRAN, respectively.

There has been a wide range of studies to measure trace species using laser spectroscopic techniques such as TDLAS, QCLAS, and CRDS [16, 26, 40-45]. However, with the most prominent exception of DOAS [46], most of them to the best of our knowledge were not referring to any documented standardized method for the spectroscopic measurements. The method of TILSAM, aiming at results that are directly traceable to the SI and which were recently made available online [29], should be viewed as an attempt to make a first step towards standardization for gas analysis applications by means of laser spectroscopy.

In this paper, we focus on the use of the TILSAM method to perform absolute concentration measurements of molecular species using different laser spectroscopic techniques. We emphasize data quality objectives expressed by means of traceability and uncertainty issues. In Section 2, we report $\mathrm{CO}_{2}$ amount fraction results performed by TDLAS based on the TILSAM method. In Section 3, similar results for $\mathrm{CO}$ performed with a quantum cascade laser operated in the intrapulsed mode are reported, and Section 4 focuses on the application of the TILSAM method to CRDS.

\section{Tunable Diode Laser Absorption Spectroscopy (TDLAS)}

In this section, we report spectrometric $\mathrm{CO}_{2}$ amount fraction measurements using TDLAS according to the TILSAM protocol [29]. The experimental setup is depicted in Figure 2. The probed transition is the $\mathrm{CO}_{2}-\mathrm{R}(12)$ line at $4987.31 \mathrm{~cm}^{-1}$ in the near infrared (NIR) combination band around $2 \mu \mathrm{m}$.

In absorption spectroscopy, the interaction of the gas species molecules at density $n$ and the sensing radiation at wave number $\tilde{v}$ is modeled by the Beer-Lambert law

$$
\Phi(\widetilde{\mathcal{v}}, L)=\Phi_{0} \cdot \exp \left\{-S_{T} \cdot r_{\text {iso }} \cdot g\left(\tilde{\nu}-\widetilde{v}_{0}\right) \cdot L \cdot n\right\}
$$

with $\Phi_{0}$ and being the incident and transmitted radiant powers, respectively, of which the SI unit is W. The absorption is governed by the molecular transition line strength $S_{T}$ at gas temperature $T$, which is in principle to be matched to the actual present isotopologue abundance by means of $r_{\text {iso }}=$ $x_{\text {isoexp }} / x_{\text {isoHIT }}$, the respective normalized absorption profile $g$ centered at $\widetilde{\nu}_{0}$, and the absorption path length $L$. The line strength

$$
\begin{aligned}
S_{T}= & S_{0}\left(\frac{Q_{T_{0}}}{Q_{T}}\right) \exp \left\{-h c \frac{E}{k_{\mathrm{B}}}\left(\frac{1}{T}-\frac{1}{T_{0}}\right)\right\} \\
& \times \frac{\left[1-\exp \left\{-h c\left(\tilde{v}_{0} / k_{\mathrm{B}} T\right)\right\}\right]}{\left[1-\exp \left\{-h c\left(\tilde{\mathcal{v}}_{0} / k_{\mathrm{B}} T_{0}\right)\right\}\right]}
\end{aligned}
$$

is specific for the probed molecular transition. $S_{0}$ is the line strength at $T_{0}=296 \mathrm{~K}$, and $Q_{T o}$ and $Q_{T}$ are the total internal partition functions of the molecule at temperature $T_{0}$ and $T$, respectively. The quantities, $c, h$, and $k_{\mathrm{B}}$, are the speed of light, the Planck and Boltzmann constants, respectively. $E$ is the ground state energy of the probed transition, and $v_{0}$ is its center wave number. For this work, we utilized the following expression for the line strength:

$$
\begin{aligned}
S_{T}= & S_{0}\left(\frac{T_{0}}{T}\right)^{j} \cdot \exp \left\{-h c \frac{E}{k_{\mathrm{B}}}\left(\frac{1}{T}-\frac{1}{T_{0}}\right)\right\} \\
& \times \frac{\left[1-\exp \left\{-h c\left(\tilde{\nu}_{0} / k_{\mathrm{B}} T\right)\right\}\right]}{\left[1-\exp \left\{-h c\left(\tilde{\mathcal{V}}_{0} / k_{\mathrm{B}} T_{0}\right)\right\}\right]} .
\end{aligned}
$$

Equation (3) is an approximation of (2) with the quantity $\left(Q_{T o} / Q_{T}\right)$ replaced by $\left(T_{0} / T\right)^{j}$, where $j$ depends on the molecular structure of the species and the transition being probed.

Relying on the ideal gas law, the molecular density $n$ of the absorbing species can be expressed in terms of its partial pressure $p_{\text {partial }}$ of the absorbing molecules and the gas temperature. The partial pressure can be related to the total pressure $p_{\text {total }}$ using the amount-of-substance fraction of the absorbing species, $x_{\text {species }}=p_{\text {partial }} / p_{\text {total }}$. Measuring $\Phi$ and $\Phi_{0}$ and probing a certain molecular transition for a known path length and measuring $p_{\text {total }}$ and $T$, leads to the amount fraction of the species

$$
x_{\text {species }}=-\ln \left(\frac{\Phi(\tilde{\nu})}{\Phi_{0}(\tilde{\nu})}\right) \frac{k_{\mathrm{B}} \cdot T}{S_{T} \cdot r_{\text {iso }} \cdot g\left(\widetilde{\nu}-\widetilde{\nu}_{0}\right) \cdot L \cdot p_{\text {total }}} .
$$

Introducing the spectral absorbance $A(\widetilde{\mathcal{V}})=-\ln \left(\Phi(\widetilde{\mathcal{V}}) / \Phi_{0}(\widetilde{\nu})\right)$, which in cases can also be called extinction, and making use of the normalization of $g$, (see, e.g., http://goldbook .iupac.org/A00028.html) (4) may also be written in its integral form

$$
\begin{aligned}
x_{\text {species }} & =\frac{k_{\mathrm{B}} \cdot T}{S_{T} \cdot r_{\text {iso }} \cdot L \cdot p_{\text {total }}} \int_{-\infty}^{\infty} A(\tilde{\nu}) \mathrm{d} \tilde{v} \\
& =\frac{k_{\mathrm{B}} \cdot T}{S_{T} \cdot r_{\text {iso }} \cdot L \cdot p_{\text {total }}} \cdot A_{\text {line }},
\end{aligned}
$$

where $A_{\text {line }}$ is the line area obtained by integration of the measured absorbance data over wave number. Direct absorption spectroscopy is described by the Beer-Lambert law, as expressed in (1). Using (5) to determine the amount fraction in a given gas mixture would deliver results that are SI traceable if all input quantities were SI traceable. An uncertainty budget for the measurement according to the ISO Guide to the expression of uncertainty in measurement (GUM) [33] can be evaluated with (5) as the model function.

For the following quantitative determinations, the isotopic composition factor $r_{\text {iso }}$ was always set to unity. This is due to the lack of a priori information on the actual isotopologue abundance present in the sample. However, this lack of information was to be transferred into to the uncertainty associated with the $r_{\text {iso }}=1$ [47].

2.1. Quantitative Amount Fraction Determination by TDLAS. A distributed feedback (DFB) tunable diode laser (TDL) emitting at $2 \mu \mathrm{m}$ was used to probe the desired $\mathrm{CO}_{2}$ transition at $4987.31 \mathrm{~cm}^{-1}$. A triangular-shaped laser current 


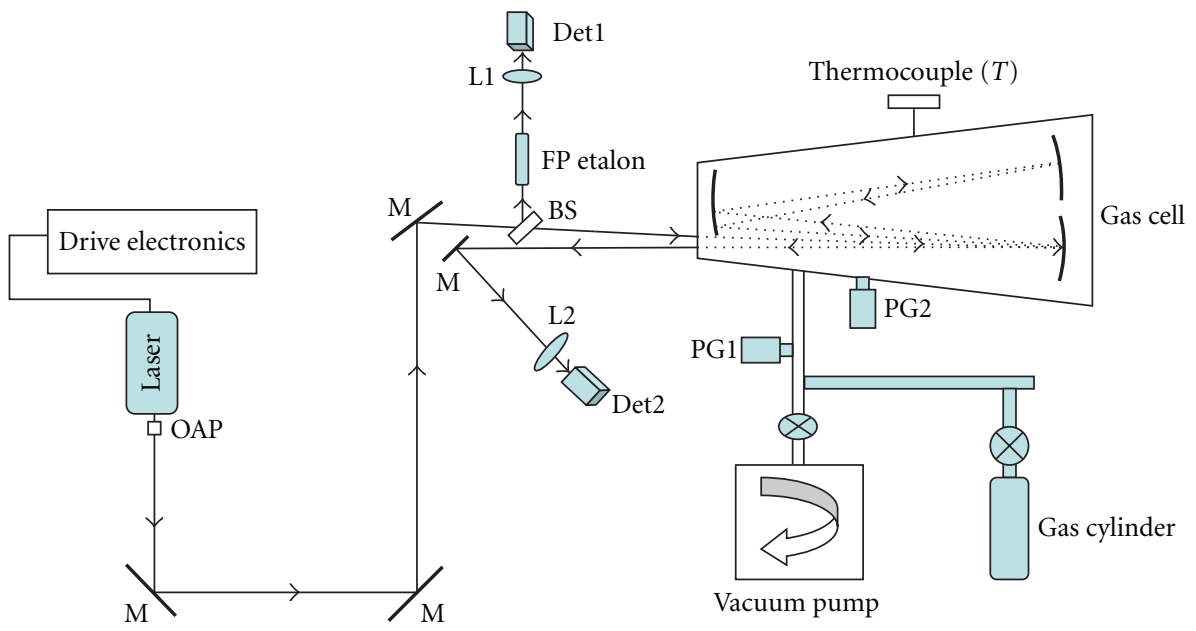

FIGURE 2: Schematic diagram of the setup used for species quantification in a two-channel regime. The multipass White-type absorption cell has a path length of $L=21 \mathrm{~m}$. OAP: off-axis parabolic mirror, BS: beam splitter, M: mirror, Det: detector, PG: pressure gauge, and L: lens.

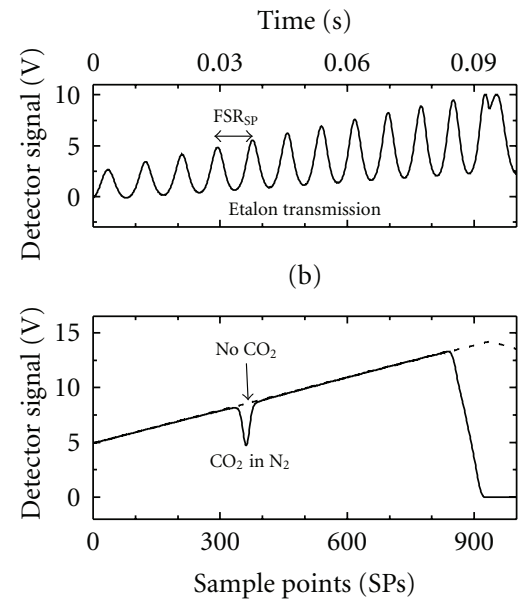

(a)

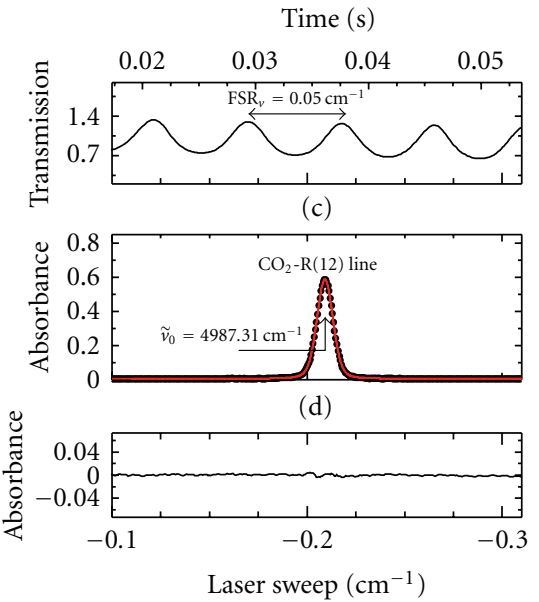

(e)

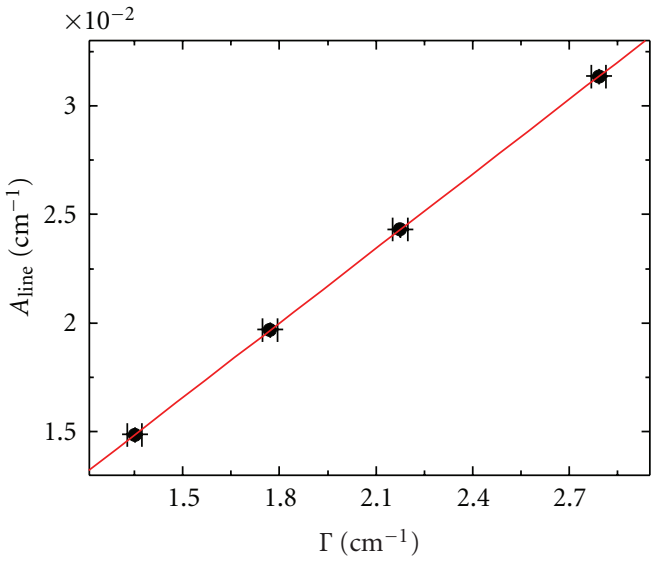

(f)

FIGURE 3: (a) Data from the reference channel, without $\mathrm{CO}_{2}$ absorption (dashed line) and the sample channel (full line) featuring $\mathrm{CO}_{2}$ absorption, respectively. (b) Etalon transmission signal seen by detector 1 with the etalon $\left(\mathrm{FSR}_{v} \approx 0.05 \mathrm{~cm}^{-1}\right)$ placed in the reference beam path. (c) and (d) Data transferred to the wave number domain. (c) Etalon transmittance curve. (d) Data of the lower panel of (a), converted to absorbance values (solid dots). A Voigt profile has been fitted to the data (full line) representing the $\mathrm{CO}_{2}-\mathrm{R}(12)$ line at $4987.31 \mathrm{~cm}^{-1}$. (e) The residuals from the fitted line in (d). (f) Generalized linear regression of $A_{\text {line }}$ versus $\Gamma$.

modulation was applied to sweep the diode laser wavelength across the $\mathrm{R}(12)$ line. After collimation, the TDL beam was split into a reference beam and a second sample beam transmitted through a $21 \mathrm{~m}$ multipass absorption cell. The reference beam was used for intensity normalization and for fixing the wavelength axis by means of a removable etalon. Both channels were terminated into extended indiumgallium-arsenide (XInGaAs) photo detectors. An A/D converter (ADLink-DG-9114) was used to digitize the electrical signals at $10 \mathrm{kS} / \mathrm{s}$.

A reference gas mixture of carbon dioxide in nitrogen, gravimetrically prepared with a nominal level of $11 \mathrm{mmol} \cdot \mathrm{mol}^{-1} \mathrm{CO}_{2}$, was filled into the gas cell for analysis. Quantitative measurements were performed based on the TILSAM method [29] with $p_{\text {total }}$ preset in the range of 10 to
$43 \mathrm{hPa}$ with the help of a gas sampling system equipped with a turbomolecular vacuum pump.

Figure 3(a) depicts the detector signals, representing a simultaneous two-channel measurement scheme. Data has been measured in the time domain in units of sample points (SPs). Figure 3(b) shows the signal of detector 1 when a Fabry Perot etalon with a free spectral range (FSR) of about $0.05 \mathrm{~cm}^{-1}$ is placed in the beam path. Figure 3(d) depicts the absorbance realized by means of the detector signals from the two channels. The residuals from the fitted $\mathrm{R}(12)$ line of $\mathrm{CO}_{2}$ are shown in Figure 3(e). The wave number axis was accomplished by means of the sweep rate, $r_{\text {sweep }}=$ $\mathrm{FSR}_{v} / \mathrm{FSP}_{\mathrm{SP}} . \mathrm{FSR}_{v}$ is the free spectral range of the etalon in wave numbers, and FSR $_{S P}$ is the experimental fringe separation measured in the time domain (SP) visible in 
Figure 3(b). The sweep rate evaluated at the absorption peak position was used to realize the wave number axis.

In Figure 3(e) a plot of the derived line area $A_{\text {line }}$ as a function of the experimental parameter $\Gamma=S_{T} \cdot L$. $p_{\text {total }} /\left(k_{\mathrm{B}} \cdot T\right)$ is shown. A variation in $\Gamma$ is mostly related to a variation of the total gas pressure. From (5), the $\mathrm{CO}_{2}$ amount fraction $\left(x_{\mathrm{CO}_{2}}\right)$ can be identified as the slope of a generalized linear regression (GLR) of values of $A_{\text {line }}$ as a function of Г. A GLR is recommended by ISO 6143 [48] for purposes like that to account for uncertainties of both, the $y$ - and the $x$-axis data. As also mentioned in ISO 6143, we applied the B_LEAST software to perform the GLR. B_LEAST is a software developed by Bundesanstalt für Materialforschung und-prüfung (BAM), which fits model functions, here that is, a linear function, to experimental data by means of a generalised least square fit. The experimental data to be fitted must contain uncertainties in the $x$ - and $y$-axis. The uncertainties of both axes data are taken into account for the GLR by B_Least. The uncertainty of the line area in Figure 3(e) (vertical bars smaller than symbol size) is due to the uncertainty of the sweep rate $r_{\text {sweep }}$ and that of the fitted line area [49]. The uncertainty of $\Gamma$ is calculated from the uncertainties of the parameters, $S_{T}, L, p_{\text {total }}, k_{\mathrm{B}}$, and $T$. The line strength used for these measurements was taken from [36] as $1.255 \cdot 10^{-21} \mathrm{~cm}^{-1} /\left(\right.$ molecule $\left.\cdot \mathrm{cm}^{-2}\right)$ with an expanded uncertainty of $\pm 1.0 \%$ relative. The $\mathrm{CO}_{2}$ amount fraction resulting from the data in Figure 3(e) was evaluated to be $(11.43 \pm 0.23) \mathrm{mmol} \cdot \mathrm{mol}^{-1}$. The uncertainty figure $( \pm 2.0 \%$, relative) is expressed as an expanded uncertainty. The deviation of the spectrometric $x_{\mathrm{CO} 2}$ result from its gravimetric reference value of $(11.38 \pm 0.16) \mathrm{mmol} \cdot \mathrm{mol}^{-1}$ is $0.4 \%$ relative, which is well covered by the uncertainty of $2.0 \%$.

\section{Quantum Cascade Laser Absorption Spectroscopy (QCLAS)}

In this section we report results on the spectroscopy of carbon monoxide $(\mathrm{CO})$ by means of pulsed quantum cascade laser spectroscopy. A detailed discussion of our pulsed QCL spectroscopy can be found in [31]. The light source is a quantum cascade laser (QCL) emitting around $4.6 \mu \mathrm{m}$, where the fundamental band of $\mathrm{CO}$ is located. The experimental setup is the same as shown in Figure 2, except that the DFB diode laser discussed in Section 2 was replaced by the QCL. Compared to the measurements published in [31], for the present work, the $21 \mathrm{~m}$ multipass cell was used. The probed transition is the CO-P(1) line at $2139.43 \mathrm{~cm}^{-1}$. The two channels in Figure 2 were terminated into TE-cooled HgCdZnTe detectors (Vigo PDI-2TE-10.6), and the electrical signals were digitized at $5 \mathrm{GS} / \mathrm{s}$ by an 8 -bit $\mathrm{A} / \mathrm{D}$ converter (CompuScope $85 \mathrm{G}$ ).

The formalism of quantum cascade laser absorption spectroscopy (QCLAS) based on the Beer-Lambert law is the same as that of TDLAS discussed in Section 2. All QCL data shown in the present work were corrected for the detector nonlinearities as pointed out in [31].

For the spectrometric QCL measurements of $\mathrm{CO}$, a gravimetrically prepared multicomponent gas mixture of $\mathrm{CO}$,
$\mathrm{CO}_{2}$, and $\mathrm{O}_{2}$ in $\mathrm{N}_{2}$ with a nominal value of $100 \mu \mathrm{mol} \cdot \mathrm{mol}^{-1}$ $\mathrm{CO}$ was filled into the $21 \mathrm{~m}$ gas cell for analysis.

Figure 4 shows typical data of the QCLAS similar to that in Figure 3. Although QCLAS is not covered by its documentary description, the spectrometric measurements were performed according to the TILSAM method [29] with the total gas pressure $p_{\text {total }}$ in the range of 100 to $300 \mathrm{hPa}$. Two methods for operating pulsed QCLs are known, the inter-pulse $[50,51]$ and the intrapulse mode $[52,53]$. A comparison of these two modes is published in $[43,54]$. For few years, also room temperature QCLs in continuous wave $(\mathrm{cw})$ mode of operation have been used to perform laser spectroscopic measurements [55-57]. However, for the present work, our QCL was operated in the intrapulse mode.

The wave number axes in Figures 4(c) and 4(d) were realized in a similar manner as those in Section 2. The CO amount fraction retrieved from the generalized linear regression performed by B_LEAST as shown in Figure 4(e) was evaluated to be $87.3 \mu \mathrm{mol} \cdot \mathrm{mol}^{-1}$. The estimated uncertainty of this $\mathrm{CO}$ amount fraction result reads $\pm 4.6 \mu \mathrm{mol} \cdot \mathrm{mol}^{-1}$ (5.2\% relative). This uncertainty figure is comparably larger than that of the TDLAS result because of the uncertainty contribution originating from the line strength. The line strength of the $\mathrm{P}(1)$ line of $\mathrm{CO}$ at $2139.4261 \mathrm{~cm}^{-1}$ was taken from HITRAN [34]. Its uncertainty is in the 2 to 5 percentage range as specified by HITRAN. For this work we used the lower level of $2 \%$ for the uncertainty evaluation, since the $\mathrm{P}(1)$ line is one of the most intense lines in this branch. However, as seen here, there is quite some room to improve the uncertainty figure of the spectrometric $\mathrm{CO}$ amount fraction to a significantly lower number, addressing the line strength contribution by first priority. The nominal CO amount fraction of the measured sample is specified as $100 \mu \mathrm{mol} \cdot \mathrm{mol}^{-1}$. A possible discrepancy between the spectrometrically derived amount faction of $(87.3 \pm$ 4.6) $\mu \mathrm{mol} \cdot \mathrm{mol}^{-1}$ and that of the nominal value could not be exactly quantified and further evaluated at the time this paper was prepared, because the sample was taken from an ongoing comparison for which exact reference values were not yet available. These QCL results were presented here, to demonstrate the application of the TILSAM method to intrapulse QCL spectrometry.

Line strength figures taken from HITRAN are not GUM compliant, that is, neither $S_{T}=296 \mathrm{~K}$ nor $S_{T}$, as discussed in Section 1 . As a consequence, our spectrometrically measured $\mathrm{CO}$ amount fraction result is not yet traceable.

QCLAS faces a couple of challenges performing amount fraction measurements in the mid infrared. Effects such as rapid passage [1] as introduced in Section 1 and some possible nonlinearity of detectors are of concern [58]. The latter was accounted for in the presented amount fraction results by applying an appropriate correction [31]. Rapid passage occurs when the laser frequency sweep through an absorption line is faster than the collisionally induced relaxation, resulting in an asymmetric or even inverted or oscillating absorption signal [1].

Rapid passage imprints can be present in QCLAS signals typically at low pressures [59]. Figure 5 displays results of a separate measurement at $5.1 \mathrm{hPa}$ total gas pressure, 


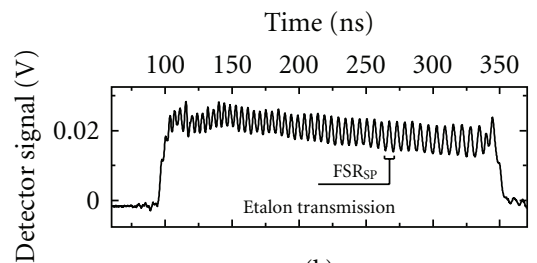

(b)

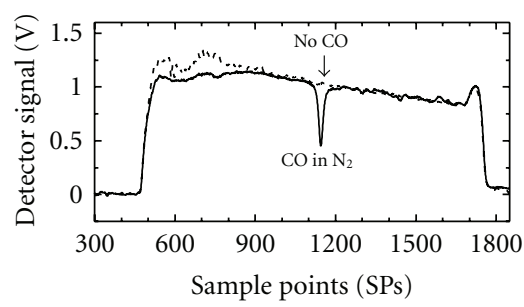

(a)

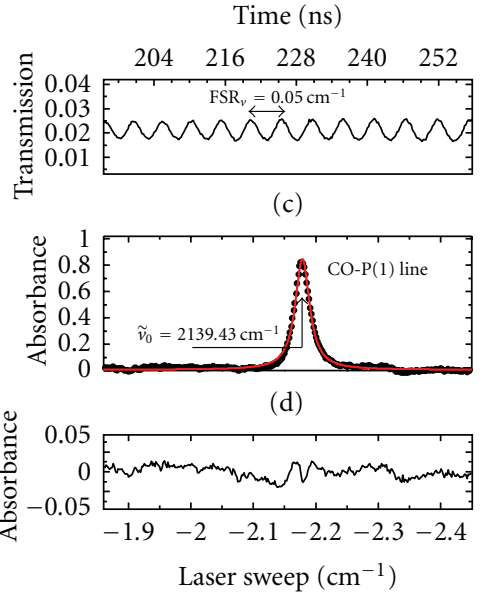

(e)

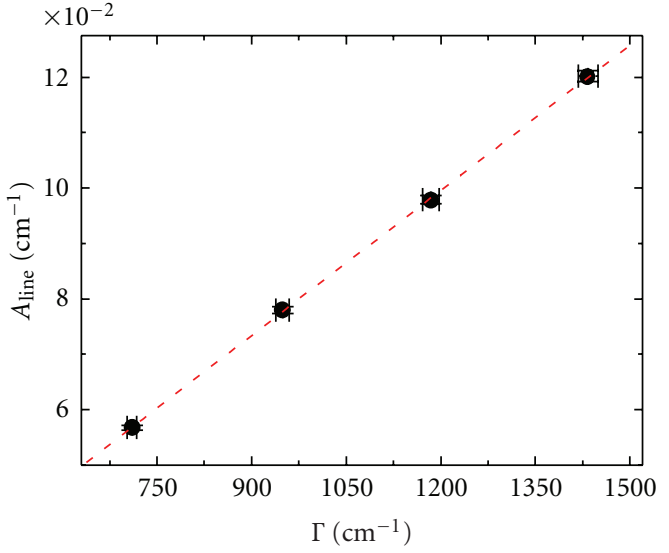

(f)

FIGURE 4: Intrapulse chirp spectroscopy. (a) Data from the reference channel, without CO absorption (dashed line) and the sample channel (full line) featuring $\mathrm{CO}$ absorption, respectively. (b) Etalon transmission signal seen by detector 1 with the etalon $\left(\mathrm{FSR}_{v} \approx 0.05 \mathrm{~cm}^{-1}\right.$ ) placed in the reference beam path. (c) and (d) Data transferred to the wave number domain. (c) Etalon transmittance curve. (d) Data of (a), converted to absorbance values. A Voigt profile has been fitted to the data (full line) representing the CO-P(1) line at $2139.43 \mathrm{~cm}^{-1}$. (e) The residuals from the fitted line in (d). QCL operating parameters: QCL substrate temperature $295 \mathrm{~K}$, pulse length $255 \mathrm{~ns}$, laser voltage $10.5 \mathrm{~V}$, repetition rate $2 \mathrm{kHz}$, QCL chip: Fraunhofer-IAF (Fraunhofer Institut für Angewandte Festkörperforschung IAF, Freiburg, Germany, http://www.iaf.fraunhofer.de/). (f) Generalized linear regression of $A_{\text {line }}$ versus $\Gamma$.

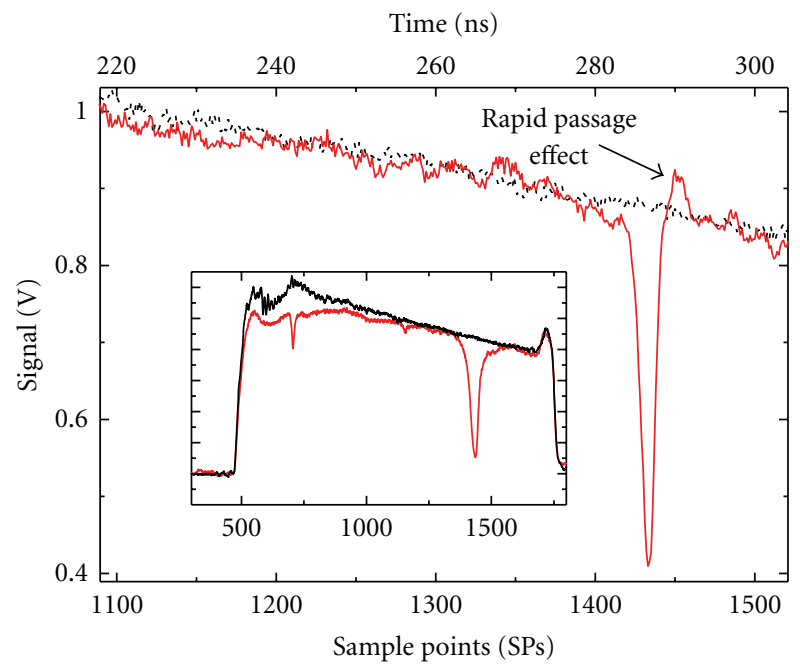

Figure 5: Rapid passage (RP) signal observed at $5.1 \mathrm{hPa}$. Inset: pressure increased to $66.5 \mathrm{hPa}$, thus depleting the RP structure, that is, no RP signal observable anymore.

performed with a gravimetrically prepared gas mixture with a nominal CO amount fraction of $1000 \mu \mathrm{mol} \cdot \mathrm{mol}^{-1}$, where rapid passage signals were observed. While increasing the pressure to $66.5 \mathrm{hPa}$, this effect was removed as visible in the inset of Figure 5. This is caused by the addition of collision partners to the absorbing medium, thus increasing the relaxation rate of the excited state molecules. Evidently, for any absolute amount fraction measurements, it is necessary to correct for such rapid passage effects or simply to eliminate them.

\section{Cavity Ring-Down Spectroscopy (CRDS)}

In this section, we report $\mathrm{CO}_{2}$ amount fraction measurements performed by CRDS referring to the TILSAM method. Different implementations of CRDS and related cavityenhanced spectroscopy (CES) techniques can be found in $[60,61]$ as well as their underlying theory. A schematic diagram of the experimental setup is shown in Figure 6. The laser source is a DFB diode laser operating around $1.6 \mu \mathrm{m}$. We probed the $\mathrm{CO}_{2}-\mathrm{R}(20)$ transition line at $6242.67 \mathrm{~cm}^{-1}$.

In CRDS the absorption coefficient of a gas sample is measured by monitoring the temporal evolution of the intensity of light trapped inside an optical cavity consisting of highly reflective mirrors. The light is reflected typically thousands of times back and forth by the mirrors. After decoupling the incoming light beam from the cavity, the buildup of light intensity inside the cavity is stopped and the intensity will decay exponentially because of various linear loss mechanisms that are present (transmission of the mirrors, absorption by the gas sample, absorption by the mirrors, diffractive losses, and scattering). The time constant of the intensity decay is called ring-down time and is denoted by the symbol $\tau$. A fast photodetector monitors the intensity of the escaping light which is proportional to the intensity of light still inside the cavity. The detected signal, therefore, also exhibits an exponential decay function from which the ring-down time is obtained.

Light intensity leaking out of the cavity is given by

$$
I(\tilde{\nu})=I_{0} \cdot \exp \left(\frac{-t}{\tau(\tilde{\nu})}\right)
$$

where $I_{0}$ is the intensity of the light inside the cavity right after decoupling the incoming light from the cavity. If one 


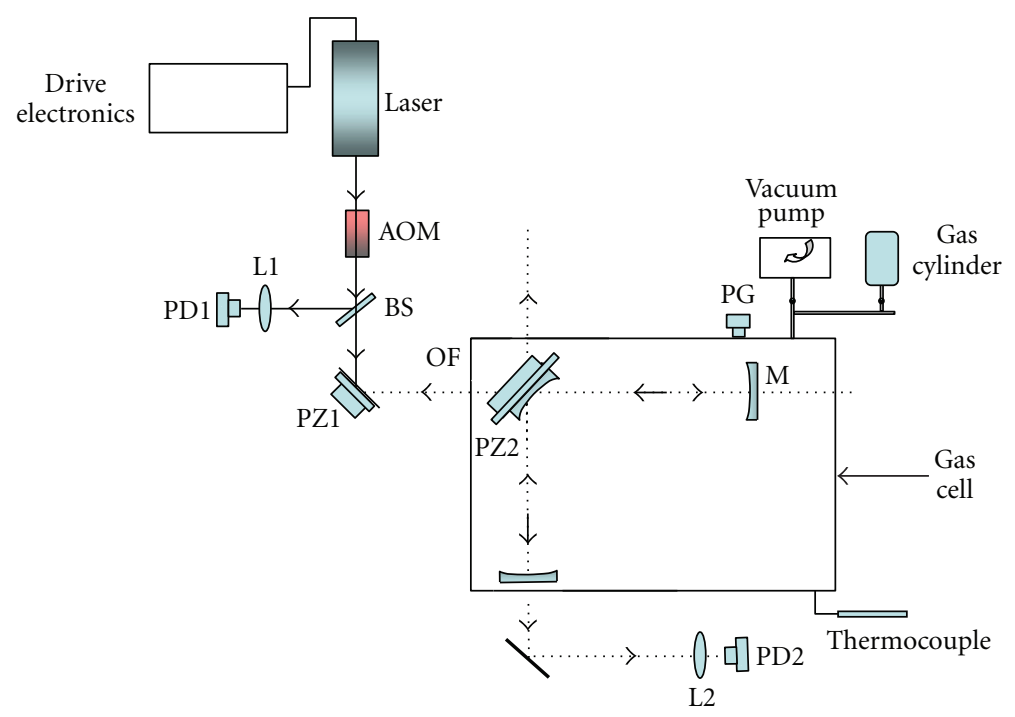

FIGURE 6: Schematic diagram of the experimental OF-CRDS setup. AOM: acousto-optical modulator, PZ: piezo-controlled mirrors, OF: optical feedback, PD: photodiode, M: mirror, PG: pressure gauge, BS: beam splitter, and L: lens.

assumes that the cell is filled homogenously with the gas sample over its entire length, then the ring-down time is given by

$$
\tau(\widetilde{\mathcal{\nu}})=\frac{L}{c\left[\left|\ln R_{\mathrm{eff}}(\widetilde{\nu})\right|+k(\widetilde{\nu}) \cdot L\right]},
$$

where $L$ is the mirror separation and $R_{\text {eff }}$ being the effective reflectivity of the mirrors including all other loss mechanisms except that of sample absorption. Note that as $R_{\mathrm{eff}} \approx 1$, the term $\left|\ln R_{\text {eff }}\right|$ is frequently replaced by $\left(1-R_{\text {eff }}\right)$. The quantity $k=S_{T} \cdot g \cdot n$ is called the absorption coefficient. $S_{T}$ is the line strength at temperature $T, g$ the normalized line profile function, and $n$ the molecular density of the absorbing species, and that might be expressed as $n=x_{\text {species }}$. $p /\left(k_{\mathrm{B}} \cdot T\right)$ as described in Section 2. Thus, the ring-down time reads as

$\tau(\widetilde{\nu})$

$$
=\frac{L}{c\left[\left|\ln R_{\text {eff }}(\tilde{\nu})\right|+S_{T} \cdot r_{\text {iso }} \cdot g\left(\tilde{\nu}-\tilde{\nu}_{0}\right) \cdot x_{\text {species }} \cdot\left(p_{\text {total }} / k_{\mathrm{B}} \cdot T\right) \cdot L\right]},
$$

where, $x_{\text {species }}$ is the amount of substance fraction, $p_{\text {total }}$ the total gas pressure, $k_{\mathrm{B}}$ the Boltzmann constant, and $T$ the gas temperature. The decay time obtained by measuring a nonabsorbing gas sample or vacuum is called $\tau_{0}$ and is given by

$$
\tau_{0}(\widetilde{\mathcal{V}})=\frac{L}{c\left[\left|\ln R_{\text {eff }}(\widetilde{\mathcal{V}})\right|\right]} .
$$

The absorption coefficient $k(v)$ is derived from the difference in the reciprocals of $\tau$ and $\tau_{0}$,

$$
\begin{aligned}
k(\widetilde{\mathcal{\nu}}) & =\frac{1}{c}\left(\frac{1}{\tau(\widetilde{\mathcal{\nu}})}-\frac{1}{\tau_{0}(\tilde{\mathcal{\nu}})}\right) \\
& =S_{T} \cdot r_{\text {iso }} \cdot \mathrm{g}\left(\tilde{\mathcal{\nu}}-\widetilde{\nu}_{0}\right) \cdot x_{\text {species }} \cdot \frac{p_{\text {total }}}{k_{\mathrm{B}} \cdot T} .
\end{aligned}
$$

By integrating both sides of (10) and rearranging them, the amount fraction of a given species can be calculated as

$$
x_{\text {species }}=A_{\text {line }} \cdot \frac{k_{\mathrm{B}} \cdot T}{S_{T} \cdot r_{\text {iso }} \cdot p_{\text {total }}},
$$

where $A_{\text {line }}$ is the line area calculated by integrating the absorption coefficient over wave number (note: in CRDS $A_{\text {line }}$ is given in units of $\mathrm{cm}^{-2}$ as compared to that of TDLAS and QCLAS given in units of $\mathrm{cm}^{-1}$ ). Equation (11) is essentially the same expression as (5), where instead the path length $L$ is included in the integral absorption coefficient $A_{\text {line }}$, allowing to obtain quantitative absorption measurements without measuring the path length. In CRDS the path length measurement is replaced by a time measurement. CRDS is restricted to the measurement of small absorptions. If absorption becomes much larger than empty cavity losses, virtually no intensity can build up in the cavity and the decay time will be correspondingly shorter. This would lead to an unreliable determination of the $\tau$ as the decay trace consists, then, of a very limited amount of data points (determined by the sampling rate of the data acquisition system).

4.1. Quantitative $\mathrm{CO}_{2}$ Amount Fraction Detection by OFCRDS. Details of the experimental setup of the optical feedback cavity-enhanced spectroscopy (OF-CES) are shown in Figure 6, and the theoretical background can be found in [62]. The spectrometer is capable of performing optical feedback absorption spectroscopy (OF-CEAS) and optical feedback cavity ring-down spectroscopy (OF-CRDS). Here, we focus on OF-CRDS or simply cavity ring down as discussed in the previous subsection. Details on how to benefit from the optical feedback performance and on the use of the different piezo-actuated mirrors displayed in Figure 6 will be addressed in an upcoming publication.

For quantitative detection of $\mathrm{CO}_{2}$, the cavity was filled with a gravimetrically prepared gas mixture with a nominal 


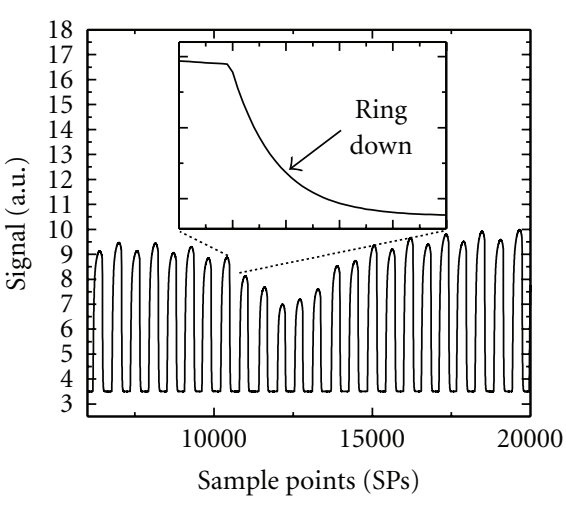

(a)

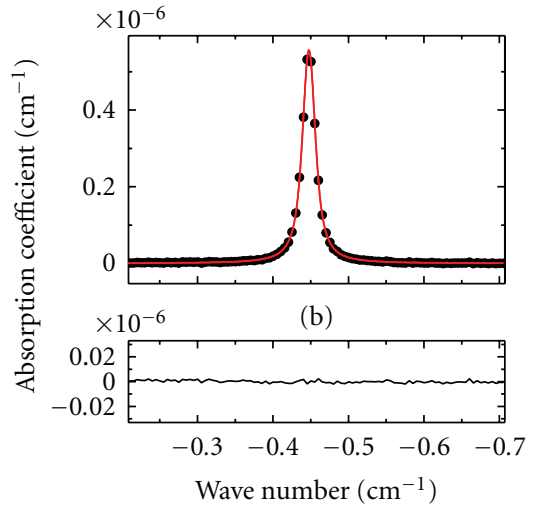

(c)

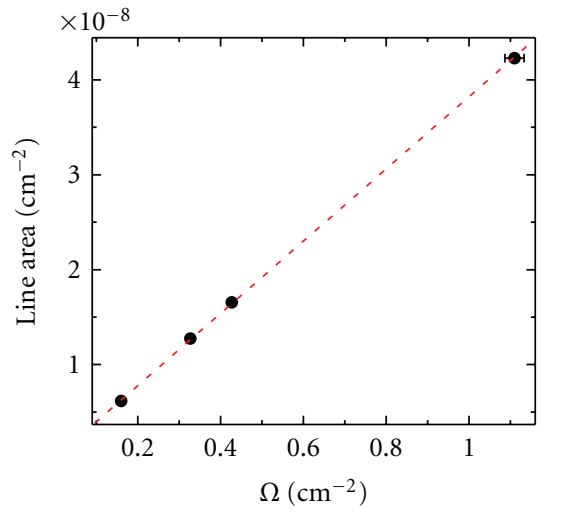

(d)

FIGURE 7: CRDS-based data retrieval: (a) typical signals from the OF-CES setup. Inset: a zoom-in of a cavity mode depicting the ring down. (b) Derived absorption coefficients fitted by a Voigt profile (full line) representing the $\mathrm{CO}_{2}-\mathrm{R}(20)$ line at $6242.6722 \mathrm{~cm}^{-1}$. (c) Residuals from the fitted line in (b). (c) Linear regression of the determined line areas $A_{\text {line }}$ versus the experimental parameter $\Omega$.

value of $389 \mu \mathrm{mol} \cdot \mathrm{mol}^{-1}$. Carbon dioxide amount fractions were measured referring to the TILSAM method to demonstrate the feasibility of applying this method also to CRDS.

Figure 7(a) depicts typical raw data, each peak representing the built-up light intensity in a certain cavity mode. In Figure 7(b), the absorption coefficient, derived from the measured ring-down times, is plotted as a function of wave number. The residuals from the fitted $\mathrm{CO}_{2}-\mathrm{R}(20)$ line are depicted in Figure $7(\mathrm{c})$. The wave number axis has been established by means of the known cavity FSR of $152 \mathrm{MHz}$ between adjacent cavity modes displayed in (a) of Figure 7. From (11), the $\mathrm{CO}_{2}$ amount fraction can be identified as the slope of a linear regression of $A_{\text {line }}$ (units: $\mathrm{cm}^{-2}$ ) versus the experimental parameter $\Omega=S_{T} \cdot p_{\text {total }} /\left(k_{\mathrm{B}} \cdot T\right)$ which is typically given in units of $\mathrm{cm}^{-2}$. A variation in $\Omega$ is mostly related to a variation of the total gas pressure.

Figure 7(c) depicts the generalized linear regression (GLR) of the derived line area values versus $\Omega$. The uncertainty of the line area is obtained from the line area fitting [49] and that of $\Omega$ is calculated from the uncertainties of the input parameters $S_{T}, p_{\text {total }}, k_{\mathrm{B}}$, and $T$. The line strength of the probed $\mathrm{R}(20)$ line of $\mathrm{CO}_{2}$ was taken from HITRAN. Its uncertainty is specified as being 2 to $5 \%$, relative.

The $\mathrm{CO}_{2}$ amount fraction resulting from the generalized linear regression, performed by B_LEAST, was evaluated to be $384 \mu \mathrm{mol} \cdot \mathrm{mol}^{-1}$ with an uncertainty of $\pm 16 \mu \mathrm{mol} \cdot \mathrm{mol}^{-1}$. The relative deviation of this spectrometric $\mathrm{CO}_{2}$ amount fraction from its nominal value of $390 \mu \mathrm{mol} \cdot \mathrm{mol}^{-1}$ was found to be $-1.5 \%$, which is well covered by the uncertainty of $\pm 16 \mu \mathrm{mol} \cdot \mathrm{mol}^{-1}( \pm 4.2 \%$ relative $)$ of the spectroscopic value. Being optimistic, the lower limit of $2 \%$ of the line strength uncertainty was taken for this uncertainty analysis knowing that the amount fraction uncertainty was to be increased once the higher limit had to be used.

\section{Discussions}

The resulting amount fractions derived by the different spectroscopic techniques are in good agreement with the respective reference values. The uncertainty evaluation is based on the method described by the ISO GUM [33].

The expanded uncertainties of the $\mathrm{CO}_{2}$ amount fraction measured with TDLAS and CRDS were found to be $\pm 2.0 \%$, and $4.2 \%$, respectively, whereas the QCLAS uncertainty analysis delivered $\pm 5.2 \%$ for the $\mathrm{CO}$ quantification. The uncertainty figures of the QCLAS and CRDS quantification are comparably larger than that of the TDLAS result. This is because the uncertainties of the line strength values used to evaluate the QCLAS and CRDS amount fraction results were in the range 2 to $5 \%$ relative, as specified by HITRAN. The $S$ value and its relative expanded uncertainty of $1.0 \%$, used to evaluate the $\mathrm{CO}_{2}$ amount fraction TDLAS result, were taken from [36]. The standard uncertainties of the other input parameters, that is, $p_{\text {total }}, k_{\mathrm{B}}, T$, and $L$, were all in the subpercentage range. Comparing the $5.2 \%$ uncertainty of the $\mathrm{CO}$ amount fraction, for instance, to the $7.5 \%$, requested by the data quality objectives set in EU directive 2000/69/EC [63] for CO field measurements, our presented results based on TILSAM would already be within this scope, although not yet validated for field measurements. However, one has to note that, if the line strength uncertainties used to derive QCLAS and CRDS amount fraction results presented in this paper were taken instead as the upper limit of $5 \%$, the relative uncertainty of the spectrometric amount of substance fraction would have to be scaled accordingly larger. Since they are in accordance, our present results, however, do support the $2 \%$ level.

A linear model developed from (5) and (11) was used to evaluate the amount fractions of $\mathrm{CO}$ and $\mathrm{CO}_{2}$ presented in this paper. The model does not predict any intercept. Therefore, as recommended in [29], the GLR was performed with a free intercept parameter. The resulting intercept parameters from the GLR are an additional measure of the quality of the measurements. The intercept parameter is either insignificant as predicted by the model or not. If the resulting intercept was significant, that is, its uncertainty is smaller than its numerical value, the model does not describe the experimental conditions appropriately. Hence, 
the quality of the spectrometric amount fraction results presented here was judged by looking at the intercept parameters resulting from the respective generalized linear regressions. The intercept parameters of the TDLAS $\mathrm{CO}_{2}$ amount fraction results calculated from the GLR were $(-5.8$ $\pm 5.8) \cdot 10^{-4} \mathrm{~cm}^{-1}$ and that of the CRDS $\mathrm{CO}_{2}$ amount fraction $(6.6 \pm 22.2) \cdot 10^{-11} \mathrm{~cm}^{-2}$, being both insignificant. A final answer could not be given at this stage for the instead significant intercept parameter of the QCLAS CO amount fraction of $(-5.2 \pm 2.6) \cdot 10^{-3} \mathrm{~cm}^{-1}$, since the measurements were performed on a sample from an ongoing comparison between different institutes, for which exact reference values are not yet known. With this result, there might be a further issue resulting from a " $w$ " structure in the residuals in Figure 4(e). This typical residual structure indicates that collisional narrowing affects the data which cannot be modeled appropriately by a Voigt profile [64]. From the oscillatory nature of the residual " $w$ " structure, it can be expected that the line area is less affected [64]. However, this proves already that by means of the TILSAM recommended data retrieval strategy, some data quality flags are provided which indicates some hidden problems with a used setup, as it is apparently now the case with our 21-m-path length QCL setup.

All the measurements presented in this paper are based on the TILSAM method as discussed earlier. The onlineavailable TILSAM documentary description [29] details the spectrometric measurement process, the raw data processing, and the uncertainty assessment principles. Although the TILSAM protocol in its present form considers only TDLAS, other techniques such as QCLAS and CRDS could be addressed by this documentary framework in a future edition as shown in this paper, Sections 3 and 4. The TILSAM method has the potential of being used in the next generation of spectrometric reference methods in gas metrology. The method could also benefit other interesting fields of application, for example, breath gas analysis, as it is aimed at in an iMERA-plus project [65], or industrial process control [66]. The TILSAM method could also be used to trigger the development of similar documentary method descriptions for the measurement of molecular reference line data, such as, for example, those of line strengths, broadening coefficients, and temperature dependencies or those of molecular constants such as spin coupling constants, ground state energy, and rotational $\mathrm{g}$ factors, or simply to support the development of process analyzers.

Contrary to measurements often performed by calibrated spectrometric systems, where the aim might be to eliminate systematic errors and study the Allan variance to provide the rms noise and the drift as a function of measurement time, our measurements performed using the TILSAM method are based on first principle measurements rather than relying on a comparison, since calibrated systems face the challenges of recalibration especially when the analyte is changed. Therefore, the use of "absolute" methods for amount fraction measurements, such as the TILSAM method, could be more cost effective than calibrated methods. Further on, calibration requires the application of reference gas mixtures. Those have to be taken aboard the spectrometric instrument by means of suitable gas containers which is not always possible due to weight and size constraints premised by some applications.

However, for the time being, the TILSAM method suffers from the unavailability of traceable line strength values and from comparably large associated uncertainties. Therefore, the line strength $S_{T}$ is the limiting factor. Traceable line strength figures are rather rare throughout the literature [39]. Nevertheless, in addition, also other quantities like the optical path length $L$ could often provide challenges if traceability is requested, for example, if multipass absorptions cells were to be considered.

Regarding the line strength, currently, there are a few efforts being undertaken to improve the lack of traceable reference line data $[36,37,65-67]$. If line strength uncertainties used in this work were in the subpercentage range, then the relative uncertainty of the spectrometric amount of substance fraction would be rigorously smaller. Consequently, the applicability would be increased and the method more competitive to existing nonspectrometric techniques. In turn, with respect to the $\mathrm{CO}$ measurements reported above, there is still some way to go before the uncertainty figure assigned to the $\mathrm{CO}$ amount fraction would match, for example, that of the data quality objective established by the WMO to be $0.5 \%$ relative for the GAW programme [68]. Thus, with an uncertainty of the amount fraction result less than one percent, the spectrometric measurements based on TILSAM presented in this paper could be applicable to environmental measurements as well as to other interesting gas analysis applications.

\section{Conclusions}

We have demonstrated the feasibility of the TILSAM method using TDLAS, QCLAS, and CRDS to perform absolute amount of substance fraction measurements. The spectrometric amount fraction results agree with their respective gravimetric reference values where available. The uncertainties of $\mathrm{CO}$ and $\mathrm{CO}_{2}$ amount fractions evaluated by TDLAS, QCLAS, and CRDS are $\pm 2.0 \%, \pm 5.2 \%$, and $\pm 4.2 \%$, respectively. We project that, with a reduced uncertainty of the line strength value, absolute spectrometric measurements based on TILSAM could be applied to multiple gas analysis applications.

\section{Acknowledgments}

Parts of this work were financially supported by ERA-NET Plus, under the iMERA-Plus Project-Grant Agreement no. 217257, by the International Graduate School of Metrology (IGSM), TU Braunschweig, and by the BMBF projects MEX07/004, and QUANSYS/QUANKAS, FKZ 13 N8123. The authors acknowledge the continuous collaboration of Professor Dr. Karl-Heinz Gericke (TU Braunschweig), Hans-Joachim Heine (BAM), and Jorge Koelliker Delgado (CENAM). Parts of this work were performed under the scientific collaboration agreement between PTB and LSP/Floralis (Université Joseph Fourier). 


\section{References}

[1] G. Duxbury, N. Langford, M. T. McCulloch, and S. Wright, "Rapid passage induced population transfer and coherences in the 8 micron spectrum of nitrous oxide," Molecular Physics, vol. 105, no. 5-7, pp. 741-754, 2007.

[2] W. Demtröder, Laser Spectroscopy: Basic Principles, vol. 1, Springer, Berlin, Germany, 4th edition, 2008.

[3] K. McCann, M. Wagner, A. Guerra et al., "Spectroscopic investigations and potential energy surfaces of the ground and excited states of 1,3-benzodioxan," Journal of Chemical Physics, vol. 131, no. 4, Article ID 044302, 2009.

[4] V. A. Kapitanov, A. M. Solodov, T. M. Petrova, and Y. N. Ponomarev, "Fourier transform and photoacoustic absorption spectra of ethylene within $6035-6210 \mathrm{~cm}^{-1}$ : comparative measurements," International Journal of Spectroscopy, vol. 2010, Article ID 203672, 6 pages, 2010.

[5] K. L. Plath, K. Takahashi, R. T. Skodje, and V. Vaida, "Fundamental and overtone vibrational spectra of gas-phase pyruvic acid," Journal of Physical Chemistry A, vol. 113, no. 26, pp. 7294-7303, 2009.

[6] M. Gharavi and S. G. Buckley, "Diode laser absorption spectroscopy measurement of linestrengths and pressure broadening coefficients of the methane $2 v_{3}$ band at elevated temperatures," Journal of Molecular Spectroscopy, vol. 229, no. 1, pp. 78-88, 2005.

[7] X. Li, K. L. C. Hunt, F. Wang, M. Abel, and L. Frommhold, "Collision-induced infrared absorption by molecular hydrogen pairs at thousands of kelvin," International Journal of Spectroscopy, vol. 2010, Article ID 371201, 11 pages, 2010.

[8] F. Huisken, M. Kaloudis, M. Koch, and O. Werhahn, "Experimental study of the $\mathrm{O}-\mathrm{H}$ ring vibrations of the methanol trimer," Journal of Chemical Physics, vol. 105, no. 19, pp. 8965$8968,1996$.

[9] F. Huisken, S. A. Krasnokutski, A. Y. Ivanov, and O. Werhahn, "The $\mathrm{O}-\mathrm{H}$ stretching vibrations of glycine trapped in rare gas matrices and helium clusters," Journal of Chemical Physics, vol. 111, no. 7, pp. 2978-2984, 1999.

[10] J. Manne, O. Sukhorukov, W. Jäger, and J. Tulip, "Pulsed quantum cascade laser-based cavity ring-down spectroscopy for ammonia detection in breath," Applied Optics, vol. 45, no. 36, pp. 9230-9237, 2006.

[11] I. Ventrillard-Courtillot, T. Gonthiez, C. Clerici, and D. Romanini, "Multispecies breath analysis faster than a single respiratory cycle by optical-feedback cavity-enhanced absorption spectroscopy," Journal of Biomedical Optics, vol. 14, no. 6, Article ID 064026, 2009.

[12] M. L. Silva, D. M. Sonnenfroh, D. I. Rosen, M. G. Allen, and A. O'Keefe, "Integrated cavity output spectroscopy measurements of nitric oxide levels in breath with a pulsed roomtemperature quantum cascade laser," Applied Physics B, vol. 81, no. 5, pp. 705-710, 2005.

[13] G. Wysocki, A. A. Kosterev, and F. K. Tittel, "Spectroscopic trace-gas sensor with rapidly scanned wavelengths of a pulsed quantum cascade laser for in situ NO monitoring of industrial exhaust systems," Applied Physics B, vol. 80, no. 4-5, pp. 617625, 2005.

[14] G. Maisons, P. Gorrotxategi Carbajo, M. Carras, and D. Romanini, "Optical-feedback cavity-enhanced absorption spectroscopy with a quantum cascade laser," Optics Letters, vol. 35, no. 21, pp. 3607-3609, 2010.

[15] B. W. M. Moeskops, H. Naus, S. M. Cristescu, and F. J. M. Harren, "Quantum cascade laser-based carbon monoxide detection on a second time scale from human breath," Applied Physics B, vol. 82, pp. 649-654, 2006.
[16] G. Wysocki, M. McCurdy, S. So et al., "Pulsed quantumcascade laser-based sensor for trace-gas detection of carbonyl sulfide," Applied Optics, vol. 43, no. 32, pp. 6040-6046, 2004.

[17] S. Kassi, M. Chenevier, L. Gianfrani, A. Salhi et al., "Looking into the volcano with a Mid-IR DFB diode laser and cavity enhanced absorption spectroscopy," Optics Express, vol. 14, no. 23, pp. 11442-11452, 2006.

[18] K. Wunderle, S. Wagner, I. Pasti et al., "Distributed feedback diode laser spectrometer at $2.7 \mu \mathrm{m}$ for sensitive, spatially resolved $\mathrm{H}_{2} \mathrm{O}$ vapor detection," Applied Optics, vol. 48, no. 4, pp. B172-B182, 2009.

[19] M. R. McCurdy, Y. Bakhirkin, G. Wysocki, and F. K. Tittel, "Performance of an exhaled nitric oxide and carbon dioxide sensor using quantum cascade laser-based integrated cavity output spectroscopy," Journal of Biomedical Optics, vol. 12, no. 3, Article ID 034034, 2007.

[20] R. Q. Iannone, S. Kassi, H. J. Jost et al., "Development and airborne operation of a compact water isotope ratio infrared spectrometer," Isotopes in Environmental and Health Studies, vol. 45, no. 4, pp. 303-320, 2009.

[21] V. Weldon, J. O'Gorman, P. Phelan, J. Hegarty, and T. Tanbun$\mathrm{Ek}$, " $\mathrm{H}_{2} \mathrm{~S}$ and $\mathrm{CO}_{2}$ gas sensing using DFB laser diodes emitting at $1.57 \mu \mathrm{m}$," Sensors and Actuators B, vol. 29, no. 1-3, pp. 101107, 1995.

[22] M. Sowa, M. Mürtz, and P. Hering, "Mid-infrared laser spectroscopy for online analysis of exhaled CO," Journal of Breath Research, vol. 4, no. 4, Article ID 047101, 2010.

[23] C. E. Miller, L. R. Brown, R. A. Toth, D. C. Benner, and V. M. Devi, "Spectroscopic challenges for high accuracy retrievals of atmospheric $\mathrm{CO}_{2}$ and the orbiting carbon observatory (OCO) experiment," Comptes Rendus Physique, vol. 6, no. 8, pp. 876887, 2005.

[24] D. Crisp, R. M. Atlas, F. M. Breon et al., "The orbiting carbon observatory (OCO) mission," Advances in Space Research, vol. 34, no. 4, pp. 700-709, 2004.

[25] R. A. Toth, C. E. Miller, L. R. Brown, V. M. Devi, and D. C. Benner, "Line strengths of ${ }^{16} \mathrm{O}^{13} \mathrm{C}^{16} \mathrm{O},{ }^{16} \mathrm{O}^{13} \mathrm{C}^{18} \mathrm{O},{ }^{16} \mathrm{O}^{13} \mathrm{C}^{17} \mathrm{O}$ and ${ }^{18} \mathrm{O}^{13} \mathrm{C}^{18} \mathrm{O}$ between 2200 and $6800 \mathrm{~cm}^{-1}$," Journal of Molecular Spectroscopy, vol. 251, no. 1-2, pp. 64-89, 2008.

[26] E. R. Crosson, K. N. Ricci, B. A. Richman et al., "Stable isotope ratios using cavity ring-down spectroscopy: determination of ${ }^{13} \mathrm{C} /{ }^{12} \mathrm{C}$ for carbon dioxide in human breath," Analytical Chemistry, vol. 74, no. 9, pp. 2003-2007, 2002.

[27] K. Heinrich, T. Fritsch, P. Hering, and M. Mürtz, "Infrared laser-spectroscopic analysis of ${ }^{14} \mathrm{NO}$ and ${ }^{15} \mathrm{NO}$ in human breath," Applied Physics B, vol. 95, no. 2, pp. 281-286, 2009.

[28] A. Predoi-Cross, C. Hnatovsky, K. Strong, J. R. Drummond, and D. Chris Benner, "Temperature dependence of self- and $\mathrm{N}_{2}$-broadeningand pressure-induced shifts in the $3 \leftarrow 0$ band of CO," Journal of Molecular Structure, vol. 695-696, pp. 269286, 2004.

[29] O. Werhahn and J. C. Petersen, Eds., TILSAM Technical Protocol, Version 1.0, PTB: Physikalisch-Technische Bundesanstalt, Braunschweig, Germany, 2010, http://www.euramet .org/fileadmin/docs/projects/934_METCHEM_Interim_Report .pdf.

[30] EURAMET-934, TILSAM-Traceable Infrared Laser Spectrometric Amount Fraction Measurement, 2008, http://www .euramet.org project no. 934.

[31] J. A. Nwaboh, O. Werhahn, and D. Schiel, "Measurement of $\mathrm{CO}$ amount fractions using a pulsed quantum-cascade laser operated in the intrapulse mode," Applied Physics B, 2010.

[32] P. Ortwein, W. Woiwode, S. Fleck et al., "Absolute diode laser-based in situ detection of $\mathrm{HCl}$ in gasification processes," Experiments on Fluids, vol. 49, no. 4, pp. 961-968. 
[33] Joint Committee for Guides in Metrology (JCGM), "Evaluation of measurement data-guide to the expression of uncertainty in measurement, GUM 1995 with minor corrections, ISO IEC Guide 98-3," JCGM 100:2008, 2008, http://www.bipm.org/en/publications/guides/gum.html.

[34] L. S. Rothman et al., "HITRAN2008," Journal of Qauntitative Spectroscopy and Radiative Transfer, vol. 110, pp. 533-572, 2009, http://www.cfa.harvard.edu/HITRAN/ .

[35] N. Jacquinet-Husson, N. A. Scott, A. Chédin et al., "The GEISA spectroscopic database: current and future archive for earth and planetary atmosphere studies," Journal of Quantitative Spectroscopy and Radiative Transfer, vol. 109, no. 6, pp. 1043-1059, 2008.

[36] G. J. Padilla-Víquez, J. Koelliker-Delgado, O. Werhahn, K. Jousten, and D. Schiel, "Traceable $\mathrm{CO}_{2}-\mathrm{R}$ (12) line intensity for laser-spectroscopy-based gas analysis near $2 \mu \mathrm{m}$," IEEE Transactions on Instrumentation and Measurement, vol. 56, no. 2, pp. 529-533, 2007.

[37] G. Casa, D. A. Parretta, A. Castrillo, R. Wehr, and L. Gianfrani, "Highly accurate determinations of $\mathrm{CO}_{2}$ line strengths using intensity-stabilized diode laser absorption spectrometry," Journal of Chemical Physics, vol. 127, no. 8, Article ID 084311, 2007.

[38] "EMRP Call 2010 Industry and Environment," 2010, http://www.emrponline.eu/call2010/srte.html .

[39] L. S. Rothman, N. Jacquinet-Husson, C. Boulet, and A. M. Perrin, "History and future of the molecular spectroscopic databases," Comptes Rendus Physique, vol. 6, no. 8, pp. 897907, 2005.

[40] C. Wang and P. Sahay, "Breath analysis using laser spectroscopic techniques: breath biomarkers, spectral fingerprints, and detection limits," Sensors, vol. 9, no. 10, pp. 8230-8262, 2009.

[41] S. Welzel, G. Lombardi, P. B. Davies, R. Engeln, D. C. Schram, and J. Röpcke, "Trace gas measurements using optically resonant cavities and quantum cascade lasers operating at room temperature," Journal of Applied Physics, vol. 104, no. 9, Article ID 093115, 2008.

[42] T. Fritsch, P. Hering, and M. Mürtz, "Infrared laser spectroscopy for online recording of exhaled carbon monoxide: a progress report," Journal of Breath Research, vol. 1, no. 1, Article ID 014002, 2007.

[43] J. Manne, W. Jäger, and J. Tulip, "Sensitive detection of ammonia and ethylene with a pulsed quantum cascade laser using intra and interpulse spectroscopic techniques," Applied Physics B, vol. 94, no. 2, pp. 337-344, 2009.

[44] S. Crunaire, J. Tarmoul, C. Fittschen, A. Tomas, B. Lemoine, and P. Coddeville, "Use of cw-CRDS for studying the atmospheric oxidation of acetic acid in a simulation chamber," Applied Physics B, vol. 85, no. 2-3, pp. 467-476, 2006.

[45] A. Foltynowicz, W. Ma, and O. Axner, "Characterization of fiber-laser-based sub-doppler NICE-OHMS for quantitative trace gas detection," Optics Express, vol. 16, no. 19, pp. 14689$14702,2008$.

[46] V. Rozanov and A. Rozanov, "Differential optical absorption spectroscopy (DOAS) and air mass factor concept for a multiply scattering vertically inhomogeneous medium: theoretical consideration," Atmospheric Measurement Techniques, vol. 3, pp. 751-780, 2010, http://www.doas-bremen.de/doas_tutorial .htm.

[47] M. Berglund and M. E. Wieser, "Isotopic compositions of elements 2009 (IUPAC Technical report)," Pure and Applied Chemistry, vol. 83, no. 2, pp. 397-410, 2011.
[48] ISO 6143: 2001, Gas analysis: comparison methods for determining and checking the composition of calibration gas mixtures, International Organization for Standardization, Geneva, Switzerland, 2001.

[49] Origin 7.5 SR6, OriginLab Cooperation, Northampton, Mass, USA, 2006.

[50] K. Namjou, S. Cai, E. A. Whittaker et al., "Sensitive absorption spectroscopy with a room-temperature distributed-feedback quantum-cascade laser," Optics Letters, vol. 23, no. 3, pp. 219223, 1998.

[51] D. D. Nelson, J. H. Shorter, J. B. Mcmanus, and M. S. Zahniser, "Sub-part-per-billion detection of nitric oxide in air using a thermoelectrically cooled mid-infrared quantum cascade laser spectrometer," Applied Physics B, vol. 75, pp. 343-350, 2002.

[52] E. Normand, M. McCulloch, G. Duxbury, and N. Langford, "Fast, real-time spectrometer based on a pulsed quantumcascade laser," Optics Letters, vol. 28, no. 1, pp. 16-18, 2003.

[53] M. T. McCulloch, E. L. Normand, N. Langford, G. Duxbury, and D. A. Newnham, "Highly sensitive detection of trace gases using the time-resolved frequency downchirp from pulsed quantum-cascade lasers," Journal of the Optical Society of America B, vol. 20, no. 8, pp. 1761-1768, 2003.

[54] B. Grouiez, B. Parvitte, L. Joly, D. Courtois, and V. Zeninari, "Comparison of a quantum cascade laser used in both cw and pulsed modes. applications to the study of $\mathrm{SO}_{2}$ lines around $9 \mu \mathrm{m}, "$ Applied Physics B, vol. 90, pp. 177-186, 2008.

[55] J. Wagner, CH. Mann, M. Rattunde, and G. Weimann, "Infrared semiconductor lasers for sensing and diagnostics," Applied Physics A, vol. 78, no. 4, pp. 505-512, 2004.

[56] A. Evans, J. S. Yu, S. Slivken, and M. Razeghi, "Continuouswave operation of $\lambda \sim 4.8 \mu \mathrm{m}$ quantum-cascade lasers at room temperature," Applied Physics Letters, vol. 85, no. 12, pp. 21662168, 2004.

[57] T. Aellen, S. Blaser, M. Beck, D. Hofstetter, J. Faist, and E. Gini, "Continuous-wave distributed-feedback quantumcascade lasers on a Peltier cooler," Applied Physics Letters, vol. 83, no. 10, pp. 1929-1931, 2003.

[58] E. Theocharous, J. Ish II, and N. P. Fox, "Absolute linearity measurements on $\mathrm{HgCdTe}$ detectors in the infrared region," Applied Optics, vol. 43, no. 21, pp. 4182-4188, 2004.

[59] S. Welzel, New Enhanced sensitivity infrared laser spectroscopy techniques applied to reactive plasmas and trace gas detection, Ph.D. thesis, Ernst-Moritz-Arndt-Universität Greifswald, Greifswald, Germany, 2009.

[60] G. Berden and R. Engeln, Eds., Cavity Ring-Down Spectroscopy: Techniques and Applications, John Wiley \& Sons, Chichester, UK, 2009.

[61] R. D. van Zee and J. Patrick Looney, "Cavity-enhanced spectroscopies," in Experimental Methods in the Physical Sciences, vol. 40, Academic Press, Amsterdam, The Netherlands, 2002.

[62] J. Morville, S. Kassi, M. Chenevier, and D. Romanini, "Fast, low-noise, mode-by-mode, cavity-enhanced absorption spectroscopy by diode-laser self-locking," Applied Physics B, vol. 80, no. 8, pp. 1027-1038, 2005.

[63] Directive 2000/69/EC of the european parliament and of the council of 16 november 2000 relating to limit values for benzene and carbon monoxide in ambient air, Official Journal of the European Communities, 2000, http://eur-lex .europa.eu/LexUriServ/LexUriServ.do?uri=OJ:L:2000:313: 0012:0021:EN:PDF.

[64] J. Henningsen and H. Simonsen, "The $\left(22^{0} 1-00^{0} 0\right)$ band of $\mathrm{CO}_{2}$ at $6348 \mathrm{~cm}^{-1}$ : Linestrengths, broadening parameters, and pressure shifts," Journal of Molecular Spectroscopy, vol. 203, no. 1, pp. 16-17, 2000. 
[65] Breath analysis as a diagnostic tool for early disease detection Joint Research Projects funded under iMERA-plus, T2.J02, 2010. http://www.euramet.org/index.php?id=1011.

[66] P. Ortwein, W. Woiwode, S. Wagner, M. Gisi, and V. Ebert, "Laser-based measurements of line strength, self and pressurebroadening coefficients of the $\mathrm{H}^{35} \mathrm{Cl} \mathrm{R}(3)$ absorption line in the first overtone region for pressures up to $1 \mathrm{MPa}$," Applied Physics B, vol. 100, no. 2, pp. 341-347, 2010.

[67] D. Lisak, D. K. Havey, and J. T. Hodges, "Spectroscopic line parameters of water vapor for rotation-vibration transitions near $7180 \mathrm{~cm}^{-1}$," Physical Review A, vol. 79, no. 5, Article ID 052507, 2009.

[68] T. Laurila, Ed., "14th WMO/IAEA meeting of experts on carbon dioxide, other greenhouse gases and related tracer measurement techniques," GAW Report 186, World Meteorological Organization (WMO), Geneva, Switzerland, 2009, http://www.wmo.int/pages/prog/arep/gaw/gaw-reports.html. 


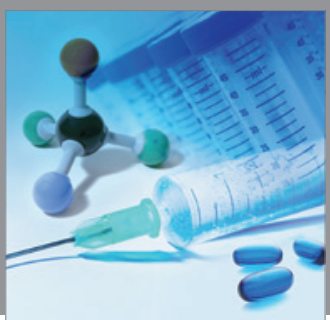

International Journal of

Medicinal Chemistry

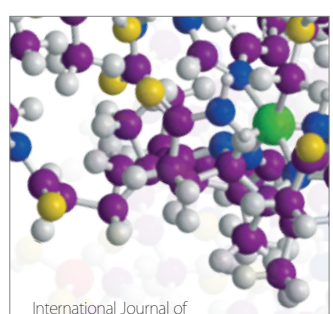

Carbohydrate Chemistry

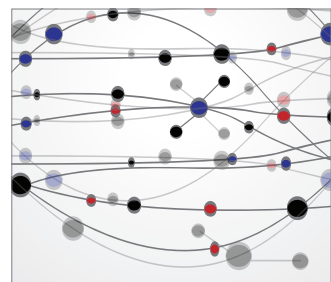

The Scientific World Journal
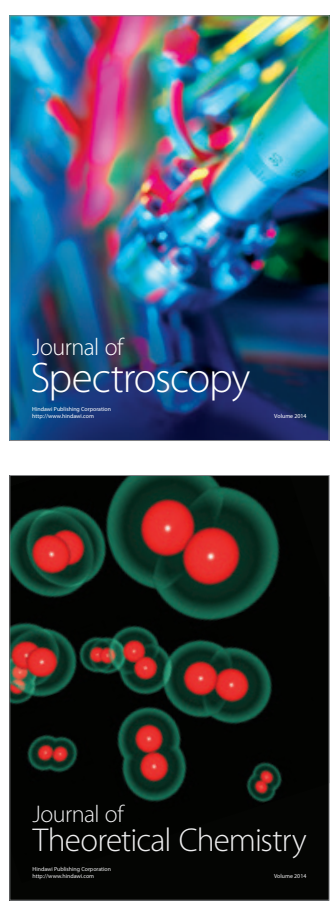
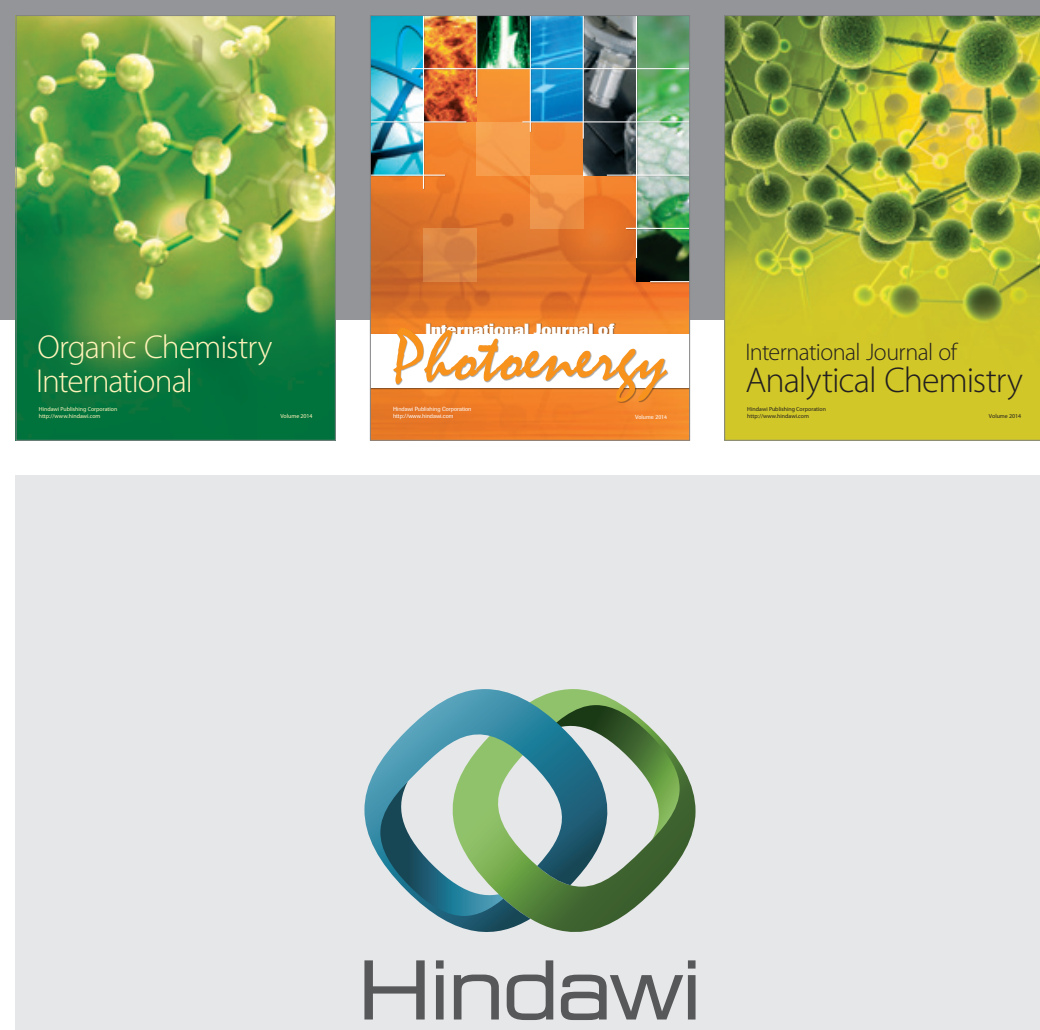

Submit your manuscripts at

http://www.hindawi.com
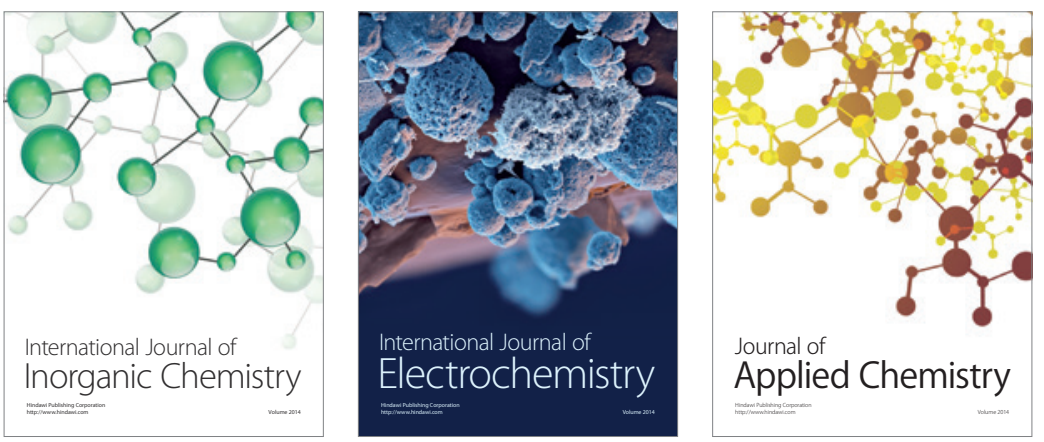

Journal of

Applied Chemistry
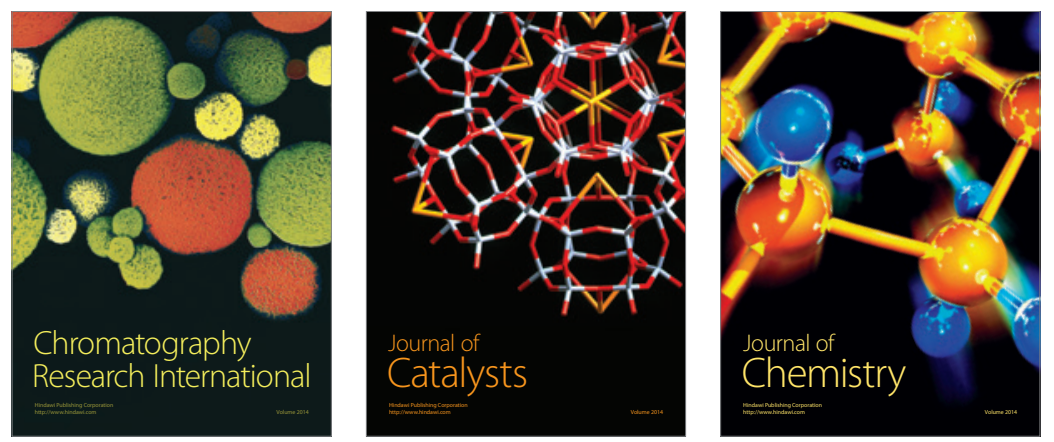
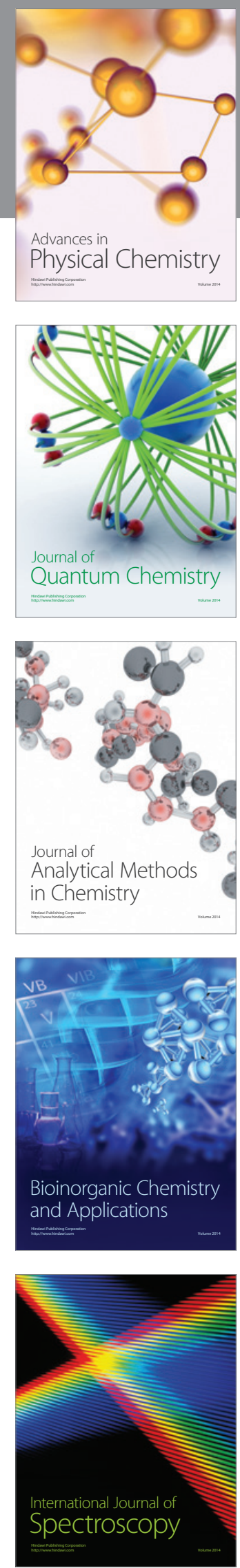